\title{
Differences between bipolar and unipolar depression on Rorschach testing
}

This article was published in the following Dove Press journal:

Neuropsychiatric Disease and Treatment

6 May 2013

Number of times this article has been viewed

\section{Hiromi Kimura \\ Akemi Osaki \\ Rui Kawashima \\ Takeshi Inoue \\ Shin Nakagawa \\ Katsuji Suzuki \\ Satoshi Asakura \\ Teruaki Tanaka \\ Yuji Kitaichi \\ Takuya Masui \\ Nobuki Kitagawa \\ Yuki Kako \\ Tomohiro Abekawa \\ Ichiro Kusumi \\ Hiroyoshi Yamanaka \\ Kenzo Denda \\ Tsukasa Koyama}

Department of Psychiatry, Hokkaido University Graduate School of

Medicine, Kita-ku, Sapporo, Japan

Correspondence: Takeshi Inoue Department of Psychiatry, Hokkaido University Graduate School of Medicine, North 15, West 7, Sapporo 060-8638, Japan

Tel +8 I I I7065 I60

Fax +811 I 1706508 I

Email tinoue@med.hokudai.ac.jp
Background: The bipolar-unipolar distinction in patients with a major depressive episode is the most important issue related to the diagnosis and treatment of mood disorders, but remains unresolved. This study was undertaken to compare bipolar and unipolar depression on Rorschach testing using the Comprehensive System with reference to healthy Japanese controls.

Methods: Patients with bipolar or unipolar depression who had undergone the Rorschach test for routine clinical purposes were followed up naturalistically for a long period. Based on diagnostic confirmation after long-term follow-up, scores on this test for patients with bipolar and unipolar depression were compared with those published elsewhere for healthy Japanese controls.

Results: The bipolar depression group showed significantly higher scores or positive findings in five variables of the Rorschach test, ie, WSum6, DR2 $>0,(\mathrm{CF}+\mathrm{C})>\mathrm{FC}+2, \mathrm{PureC}>1$, and Populars $>7$, as assessed using the Comprehensive System, than did the unipolar depression group and healthy controls. These scores did not differ between the unipolar depression and control groups.

Conclusion: The results of this study show thought disorder or cognitive slippage and marked laxness in modulating emotion in bipolar depression, indicating the psychopathological characteristics of bipolar disorder.

Keywords: bipolar depression, bipolar disorder, Rorschach test, thought disorder, unipolar depression

\section{Introduction}

The bipolar-unipolar distinction in patients with a major depressive episode is crucial for diagnosis and treatment of mood disorders. The index episode in two thirds of bipolar patients is a depressive one, ${ }^{1}$ and unipolar-to-bipolar conversion in most bipolar patients with depressive onset occurs within 5-9 years. ${ }^{2}$ Therefore, major depressive disorder is likely to include false unipolar depression, ie, unrecognized bipolar disorder, the prevalence of which is about $10 \%{ }^{2}$ Because the current guidelines for major depressive disorder and bipolar disorder differ greatly, ${ }^{3-5}$ misdiagnosis should be avoided. Introduction of antidepressant therapy without a mood stabilizer may cause manic switch and rapid cycling. Early diagnosis of unrecognized bipolar disorder before the first manic episode is necessary.

Clinical symptoms, natural course, personality, and genetics might differentiate bipolar depression from unipolar depression. Family history of bipolar disorder in a first-degree relative is the most reliable predictor of bipolarity aside from occurrence and recognition of manic episodes. ${ }^{6}$ However, differential diagnosis of bipolar and unipolar depression remains difficult clinically. Several investigators have attempted 
to discriminate bipolar-unipolar using biological tests. ${ }^{6}$ Among such studies, serotonin-induced platelet intracellular calcium mobilization ${ }^{7}$ and increased cerebral blood flow in the frontal lobe assessed using multichannel near-infrared spectroscopy ${ }^{8}$ showed clear differences between bipolar and unipolar depression. In addition, self-questionnaires for temperament and personality, such as the TEMPS-A (Temperament Evaluation of Memphis, Pisa, Paris and San Diego-autoquestionnaire version) and MMPI (Minnesota Multiphasic Personality Inventory), might be useful., ${ }^{9,10}$

The Rorschach test is a sensitive performance-based personality assessment instrument that has been used in psychiatric practice for nearly a century, and has been introduced as a standard battery in the American Psychiatric Association textbook. ${ }^{11}$ There have been controversies over the validity of the Rorschach test. For example, use of Comprehensive System normative data as a control group as reported by Exner, nonblinded judgments of coding, poor interrater reliability, selective reporting of results, and failure to control alpha level, have been criticized. ${ }^{12}$ Only two studies using the Rorschach test for comparison of bipolar depression with unipolar depression have been reported in the relevant literature. ${ }^{13,14}$ The reports describe more responses based on external characteristics of a blot, more color-naming responses, and more cognitive slippage by subjects with bipolar depression than by those with unipolar depression. However, these studies had some drawbacks, ie, the possibility exists that major depressive disorder unintentionally included latent bipolar disorder because periods from the onset of mood disorder to the final observation were not described, and the studies lacked a healthy control group that would have determined which of the unipolar or bipolar groups differs from healthy controls. Furthermore, because one study did not use the Comprehensive System developed by Exner, ${ }^{15-17}$ which is widely used as an interpretation and quantitative method of the Rorschach test now, it is not possible to compare their data with those of other studies. ${ }^{14}$

The aim of this study was to compare bipolar and unipolar depression in the Rorschach test using the Comprehensive System developed by Exner, ${ }^{15-17}$ with reference to data from a healthy control group in Japan. ${ }^{18}$ No reported study has compared these three groups using the Rorschach test. The Comprehensive System might be appropriate as an examination for differential diagnosis of unipolar and bipolar depression if results of the Rorschach test differ between those of people with bipolar and unipolar depression. In these patients, periods from the onset of mood disorder to the final observation were 5 years or longer. Therefore, diagnosis of unipolar or bipolar disorder was confirmed in our subjects to some extent, although not completely. In addition, to limit the drawbacks of the Rorschach test mentioned previously, coding and scoring were blind, and two groups of bipolar and unipolar depression were statistically compared.

\section{Materials and methods Subjects}

This study was a naturalistic retrospective study of adult patients with major depressive disorder (unipolar depression group, $\mathrm{n}=20$ ), bipolar I disorder (most recent episode depressed, $n=10)$, or bipolar II disorder $(n=10)$, (the latter two designated as bipolar depression group), who had been treated at the Department of Psychiatry, Hokkaido University Hospital. Diagnoses of mood disorder were made in accordance with the DSM-IV (Diagnostic and Statistical Manual of Mental Disorders, 4th Edition) by experienced psychiatrists responsible for these patients. ${ }^{19}$ The patients underwent Rorschach testing during 1996-2005 when their depression had become slight to moderate with pharmacotherapy. The severity of depression was evaluated at the time of testing using the Clinical Global Impression scale. At the time of testing, the results of this test were used clinically for the understanding of personality, but did not affect the bipolarunipolar distinction. They had a total intelligence quotient of at least 70 on the WAIS-R (Wechsler Adult Intelligence Scale-Revised). Depressed patients with brain magnetic resonance imaging or electroencephalographic evidence of organic brain disease or a concurrent severe medical problem were excluded from this study. Patients who were diagnosed with bipolar spectrum disorder ${ }^{20}$ or substance-induced mood disorder ${ }^{19}$ were excluded from the unipolar depression group. These patients were followed up until December 2007. Their diagnoses were confirmed during the follow-up period, which was 5 years or more from onset of their mood disorder, given that much bipolar conversion takes place during the first 5 years. ${ }^{2}$ This study was performed in accordance with the Declaration of Helsinki and approved by the institutional review board of Hokkaido University Hospital.

\section{Rorschach test}

The full Rorschach test (10 blots) was ordered by attending psychiatrists for diagnosis and treatment in routine clinical work. The test was administered to the participants by clinical psychologists. Both the administration and inquiry phases of the test were conducted in accordance with standard procedures as outlined in the Comprehensive System by Exner. ${ }^{15-17}$ All test responses were handwritten 
by the examiner and recorded on one location sheet per participant using the Structural Summary Blank published by Rorschach Workshops. Standard seating procedures were followed. ${ }^{17}$ Scoring of protocols were conducted individually in accordance with the most current available version of the Comprehensive System ${ }^{16}$ by two expert clinical psychologists (HK, AO, or RK) who had been trained extensively in the Comprehensive System and were blinded to each subject's identity and disease status. The coding of each was reviewed. Substantial coding disagreement was discussed and resolved between the senior reviewers. The protocol was not included in the sample if the total responses (R) were fewer than 14 . Scores on this test for the unipolar depression and bipolar depression groups were compared with those of the healthy Japanese controls reported by Nakamura et al. ${ }^{18}$

\section{Data analyses}

Continuous data are presented as the mean \pm standard deviation. Statistical analyses were done using JMP 8 software (SAS Institute Inc, Cary, NC, USA), and GraphPad Prism 4, (GraphPad Software, La Jolla, CA, USA). For dichotomous variables, the Kruskal-Wallis test between the three groups (unipolar depression, bipolar depression, and controls) was used to calculate the $P$ values followed by Dunn's multiple comparison test. For all other continuous variables, if the homogeneity of variance was confirmed by Levene's test, one-way analysis of variance and the Tukey-Kramer post hoc test were used. However, if the heterogeneity of variance was confirmed by Levene's test, then Welch's analysis of variance and Scheffe's post hoc test were used.

Univariate logistic regression analysis was used to screen variables that were significantly associated with a bipolar diagnosis. Multivariate logistic regression analysis was performed using features that were significant in the univariate analysis to identify independent factors for a bipolar or unipolar diagnosis and to control for confounding and interactions. Excel Statistics for Macintosh (Esumi Co, Ltd, Tokyo, Japan) was used for logistic regression analysis. Differences were considered to be statistically significant at $P<0.05$. Inter-rater reliability $(\kappa)$ was analyzed for various variables.

\section{Results}

\section{Clinical and patient data}

Patient clinical and demographic characteristics for the unipolar depression and bipolar depression groups are shown in Table 1. The interval between onset of the mood disorder to Rorschach testing and the final observation was longer in the bipolar depression group than in the unipolar depression group. Duration from onset to the final observation was adequate in both groups to confirm

Table I Clinical and demographic characteristics for the bipolar depression, unipolar depression, control subjects

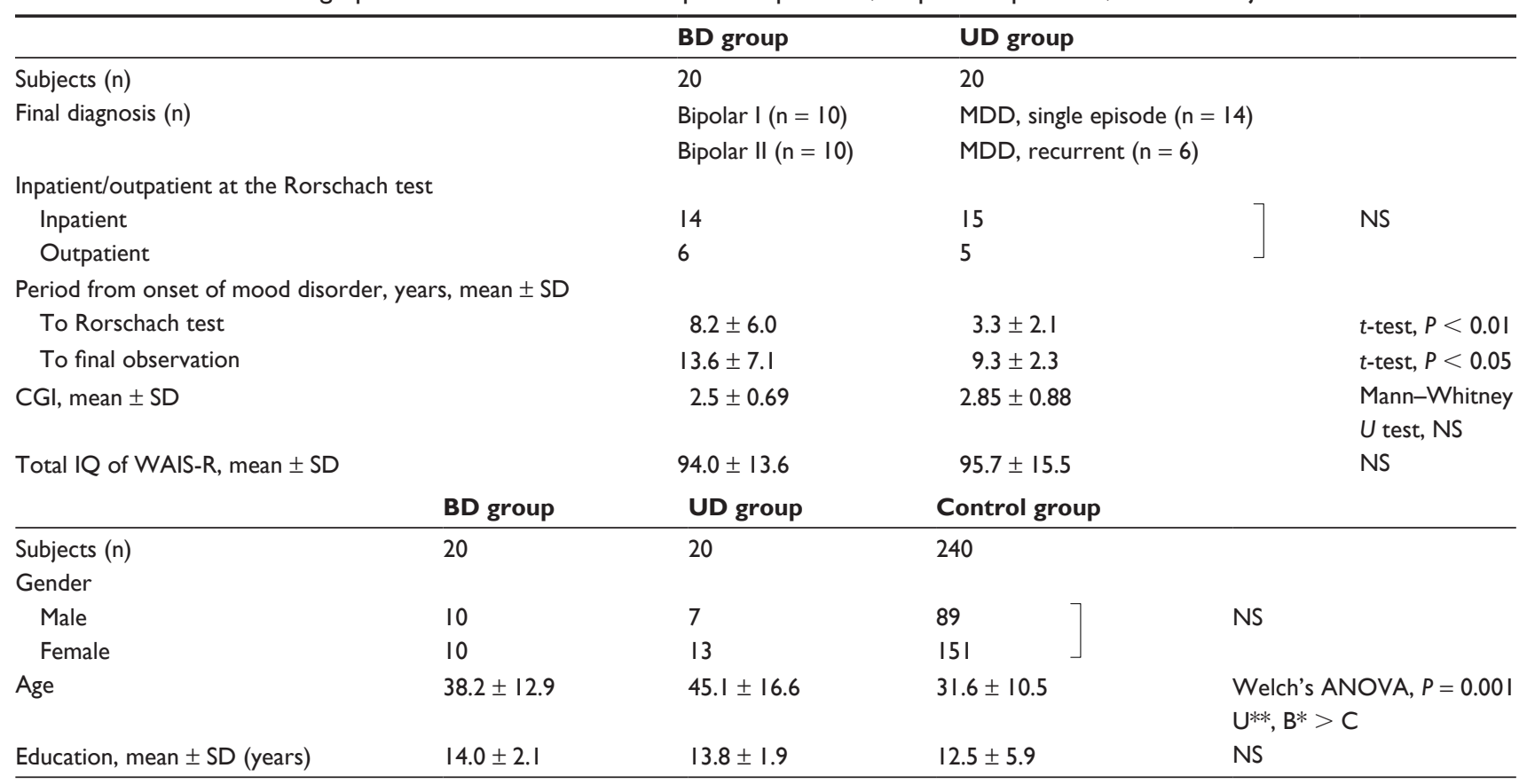

Notes: $* * p<0.01 ; * p<0.05$.

Abbreviations: ANOVA, analysis of variance; NS, not significant; BD, bipolar depression; IQ, intelligence quotient; MDD, major depressive disorder; WAIS-R, Wechsler Adult Intelligence Scale-Revised; CGI, Clinical Global Impression scale (I, normal; 2, slightly ill; 3, mildly ill; 4, moderately ill; 5, markedly ill; 6, severely ill; 7, extremely ill); $\mathrm{SD}$, standard deviation; B, BD, bipolar depression; U, UD, unipolar depression; C, control. 
bipolarity (average $>9$ years). Depressive symptoms were slight to moderate in both groups. Further, the severity of the Clinical Global Impression score was not different between the two groups. Compared with the control group, subjects with bipolar depression or unipolar depression were older, but gender ratios and education did not differ (Table 1).

\section{Rorschach data}

$\kappa$ coefficients for inter-rater reliability were 0.86 for whole responses, 0.92 for location and space (two variables), 0.83 for DQ $(+, \mathrm{o}, \mathrm{v} /+, \mathrm{v}), 0.88$ for determinants (11 variables),
0.73 for FQ (+, o, u, - , none), 0.92 for pairs, 0.86 for content (27 variables), 0.94 for popular, 0.83 for $\mathrm{Z}$ score, and 0.79 for special scores (14 variables). Inter-rater reliability in this study was excellent for most variables, except for special scores, and was comparable with that in a previous study. ${ }^{18}$

The average total number of responses $(\mathrm{R})$ did not differ between groups (Table 2) although, if significant, it might affect the results of other analyses. The statistical analyses of continuous and dichotomous variables of the Rorschach test are shown in Tables 3-5. None of the special indices (S-CON, PTI, DEPI, CDI, HVI, and OBS) differed between the groups (data not shown).

Table 2 Descriptive statistics and $F$ values for continuous variables of the Rorschach test

\begin{tabular}{|c|c|c|c|c|c|c|}
\hline & $\begin{array}{l}\text { BD } \\
\text { Mean (SD) }\end{array}$ & $\begin{array}{l}\text { UD } \\
\text { Mean (SD) }\end{array}$ & $\begin{array}{l}\text { Control } \\
\text { Mean (SD) }\end{array}$ & $\boldsymbol{F}$ & $P$ & Post hoc \\
\hline $\mathrm{R}$ & $25.10(11.00)$ & 20.70 (9.30) & $26.25(9.97)$ & 2.89 & 0.057 & Not done \\
\hline \multicolumn{7}{|l|}{ Controls } \\
\hline M & $4.05(3.35)$ & $3.65(3.08)$ & $5.62(3.14)$ & 5.49 & 0.005 & $\mathrm{U}^{*}<\mathrm{C}$ \\
\hline Sum Shd & $2.10(1.71)$ & $2.40(2.96)$ & $3.70(2.88)$ & 4.58 & 0.011 & $\mathrm{~B}^{*}<\mathrm{C}$ \\
\hline EA & $8.50(6.09)$ & $6.05(3.63)$ & $8.98(4.20)$ & 4.28 & 0.015 & $\mathrm{U}^{*}<\mathrm{C}$ \\
\hline es & $7.40(5.27)$ & $6.60(4.08)$ & 9.11 (4.50) & 3.88 & 0.022 & $\mathrm{U}^{*}<\mathrm{C}$ \\
\hline \multicolumn{7}{|l|}{ Affect } \\
\hline Blends & $3.20(3.12)$ & $2.65(2.43)$ & $4.32(3.03)$ & 3.88 & 0.022 & $\mathrm{U}^{*}<\mathrm{C}$ \\
\hline \multicolumn{7}{|l|}{ Processing } \\
\hline $\mathrm{D}$ & $8.55(5.08)$ & $6.45(3.86)$ & $9.97(6.00)$ & 3.74 & 0.025 & $\mathrm{U}^{*}<\mathrm{C}$ \\
\hline DQ+ & $5.90(4.83)$ & $5.10(2.92)$ & $7.83(3.57)$ & 7.30 & 0.001 & $\mathrm{U}^{* *}<\mathrm{C}$ \\
\hline $\mathrm{Zf}$ & $16.20(8.04)$ & I2.75 (5.2I) & I6.16 (5.7I) & 3.14 & 0.045 & $\mathrm{U}^{*}<\mathrm{C}$ \\
\hline \multicolumn{7}{|l|}{ Mediation } \\
\hline FQo & II.95 (5.34) & $9.40(2.4 I)$ & I2.88 (4.2I) & 6.57 & 0.002 & $\mathrm{U}^{* *}<\mathrm{C}$ \\
\hline \multicolumn{7}{|l|}{ Ideation } \\
\hline MQo & $2.70(2.00)$ & 2.05 (1.79) & 3.57 (1.99) & 6.80 & 0.001 & $\mathrm{U}^{* *}<\mathrm{C}$ \\
\hline $\mathrm{Ma}$ & $2.00(2.58)$ & $\mathrm{I} .45(\mathrm{I} .50)$ & $2.75(2.14)$ & 4.28 & 0.015 & $\mathrm{U}^{*}<\mathrm{C}$ \\
\hline active & $4.80(4.88)$ & $3.15(2.4 \mathrm{I})$ & $5.32(3.14)$ & 4.23 & 0.015 & $\mathrm{U}^{*}<\mathrm{C}$ \\
\hline Sum6 & $5.35(3.87)$ & $3.35(2.58)$ & $2.97(2.85)$ & 6.20 & 0.002 & $\mathrm{~B} * *>\mathrm{C}$ \\
\hline WSum6 & $14.40(12.25)$ & 7.65 (7.34) & $8.19(8.69)$ & 4.62 & 0.011 & $\mathrm{~B}>\mathrm{U}^{*}, \mathrm{C}^{* *}$ \\
\hline \multicolumn{7}{|c|}{ Self-perception and interpersonal perception } \\
\hline $\mathrm{Hd}$ & $0.85(0.93)$ & $1.45(1.54)$ & $2.05(2.00)$ & 4.27 & 0.015 & $\mathrm{~B}^{*}<\mathrm{C}$ \\
\hline GHR & $4.15(2.46)$ & $3.50(1.64)$ & $5.01(2.39)$ & 4.74 & 0.009 & $\mathrm{U}^{*}<\mathrm{C}$ \\
\hline \multicolumn{7}{|l|}{ Others } \\
\hline $\mathrm{Cg}$ & $\mathrm{I} .40(2.70)$ & $1.10(1.29)$ & $3.13(2.35)$ & 11.34 & 0.000 & $\mathrm{~B}, \mathrm{U}<\mathrm{C}^{* *}$ \\
\hline $\mathrm{Hh}$ & $1.35(1.84)$ & $0.80(1.01)$ & $0.70(1.06)$ & 3.08 & 0.047 & $B^{*}>C$ \\
\hline Idio & $0.40(0.99)$ & $0.65(0.75)$ & $1.36(1.49)$ & 6.07 & 0.003 & $\mathrm{~B}^{*}<\mathrm{C}$ \\
\hline
\end{tabular}

Notes: The following variables were not statistically significant between groups: (Controls) R, FM, m, F, Lambda, FM+ m, D Score, AdjD; (Affect) FC, CF, Sum Color, WSumC, Blend\%, Afr; (Processing) W, DQo, Zd; (Mediation) FQ+, FQu, FQ-, Popular, XA\%, WDA\%, X+\%, X-\%, Xu\%, PureF\%; (Ideation) MQ+ MQu, Mp, Passive, Intellect, Art;

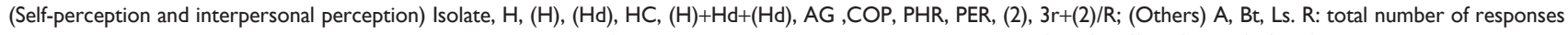
to any location in the blot. M: human movement, a determinant reflecting kinesthesis or activity involving humans. Sum Shd (Sum Shading): All of the shading and achromatic determinants in a record. EA: Experience Actual, the addition of Sum M and WSumC (weighted sum color). ES: Experienced Stimulation, the sum of FM + m (addition of all animal movement and all inanimate, inorganic, or insensate movement) and Sum Shd. Blends: A response that contains two or more determinants, eg, "a person with a black (ie, achromatic) hat is dancing (ie, human movement)". D: location code indicating a response involving a major detail, one identified in the Card's location table. DQ+: a developmental quality code reflecting a synthesized answer. Zf: The total number of Z scores (organizational activity) in a record. FQo: All the Form Quality ordinary in a record. MQo: All the Form Quality ordinary for Human Movement (M) in a record. Ma: human movement that is active. Active: superscript for movement determinants denoting a higher level of behavioral output than $\mathrm{P}$ (passive). Sum6: All of the cognitive special scores in a record (DV, INC, DR, FAB, ALOG, and CONTAM), which are described in the footnotes of Table 3. WSum6: weighted Sum6, the weighted sum of the six cognitive special scores (DV, INC, DR, FAB, ALOG, and CONTAM). Hd: content code for reference to a real human detail. GHR: good human representational response. Cg: content code for the use of clothing. Hh: content code for reference to household items. Idio (idiographic): content that is not captured by other content categories. $* * P<0.01$; $* P<0.05$. Adapted with permission Glossary of Comprehensive System Scores. J Pers Assess. 2007;89 Suppl I:S217-S220.22

Abbreviations: B, BD, bipolar depression; U, UD, unipolar depression; C, control; SD, standard deviation. 
Table 3 Descriptive statistics and $P$ values for dichotomous variables of six cognitive special scores, from which WSum6 is calculated $[\mathrm{n}(\%)]$

\begin{tabular}{|c|c|c|c|c|c|}
\hline & $B D(n=20)$ & UD $(n=20)$ & Control $(n=240)$ & $\begin{array}{l}\text { Kruskal-Wallis } \\
\text { test, } P\end{array}$ & Post hoc \\
\hline $\mathrm{DVI}>0$ & $12(60 \%)$ & $10(50 \%)$ & $65(27 \%)$ & 0.002 & $\mathrm{~B}^{* *}>\mathrm{C}$ \\
\hline $\mathrm{DV} 2>0$ & I (5\%) & $0(0 \%)$ & $\mathrm{I}(0 \%)$ & 0.061 & \\
\hline $\mathrm{INCI}>0$ & 15 (75\%) & 17 (85\%) & $15 \mid(63 \%)$ & 0.089 & \\
\hline $\mathrm{INC2}>0$ & I (5\%) & I (5\%) & $17(7 \%)$ & 0.889 & \\
\hline$D R I>0$ & $12(60 \%)$ & $5(25 \%)$ & $54(23 \%)$ & 0.001 & $\mathrm{~B}^{* *}>\mathrm{C}$ \\
\hline $\mathrm{DR} 2>0$ & $6(30 \%)$ & I (5\%) & $4(2 \%)$ & 0.000 & $\mathrm{~B}>\mathrm{U}^{* *}, \mathrm{C}^{* *}$ \\
\hline $\mathrm{FABI}>0$ & $8(40 \%)$ & $6(30 \%)$ & $108(45 \%)$ & 0.408 & \\
\hline $\mathrm{FAB} 2>0$ & $0(0 \%)$ & $0(0 \%)$ & II (5\%) & 0.386 & \\
\hline ALOG $>0$ & I (5\%) & $0(0 \%)$ & $19(8 \%)$ & 0.389 & \\
\hline CONT $>0$ & $0(0 \%)$ & $0(0 \%)$ & $2(1 \%)$ & 0.846 & \\
\hline
\end{tabular}

Notes: Some variables are subdivided to level I and level 2. Level 2 is more severe than level I. DV, Deviant Verbalization, use of a neologism, an individualized meaning, or redundancy; INC (INCOM), Incongruous Combination, attribution of some aspect or activity to a response that is out of keeping with that response; DR, Deviant Response, use of an inappropriate phrase or circumstantiality; FAB (FABCOM), Fabulized Combination, use of an implausible relationship; ALOG, Autistic Logic, use of strained reasoning; CONT (CONTAM), Contamination, the merging or blending or both of two contents within one blot area. $* * P<0.01$; $* P<0.05$. Adapted with permission Glossary of Comprehensive System Scores. J Pers Assess. 2007;89 Suppl I:S2 17-S220.22

Abbreviations: B, BD, bipolar depression; U, UD, unipolar depression; C, control.

Of the ideation cluster, MQo, Ma, and active scores in the unipolar depression group were significantly lower than those of controls. The Sum6 score in the bipolar depression group was significantly higher than that in controls (Table 2). In addition, Lv2 $>0$ was more frequent in the bipolar depression group than in controls (Table 4).
However, these parameters were not different between the bipolar depression and unipolar depression groups. The weighted sum of the six cognitive special scores (DV, INC, DR, FAB, ALOG, and CONT), as shown in Table 3. WSum6 in the bipolar depression group differed significantly from that in the unipolar depression group and in

Table 4 Descriptive statistics and $P$ values for dichotomous variables of the Rorschach test [n, (\%)]

\begin{tabular}{|c|c|c|c|c|c|}
\hline & $B D(n=20)$ & UD $(n=20)$ & Control $(n=240)$ & $\begin{array}{l}\text { Kruskal-Wallis } \\
\text { test, } P\end{array}$ & Post hoc \\
\hline \multicolumn{6}{|l|}{ Controls } \\
\hline Extratensive & $5(25 \%)$ & I (5\%) & $16(7 \%)$ & 0.012 & $\mathrm{~B}^{*}>\mathrm{C}$ \\
\hline \multicolumn{6}{|l|}{ Affect } \\
\hline$(C F+C)>F C+2$ & $8(40 \%)$ & $2(10 \%)$ & $40(17 \%)$ & 0.021 & $\mathrm{~B}>\mathrm{U}^{*}, \mathrm{C}^{*}$ \\
\hline PureC $>1$ & $5(25 \%)$ & $0(0 \%)$ & $14(6 \%)$ & 0.002 & $\mathrm{~B}>\mathrm{U}^{* *}, \mathrm{C}^{* *}$ \\
\hline \multicolumn{6}{|l|}{ Mediation } \\
\hline WDA $\%<0.85$ & $18(90 \%)$ & $12(60 \%)$ & I 48 (62\%) & 0.038 & $\mathrm{~B}^{*}>\mathrm{C}$ \\
\hline Populars $>7$ & $6(30 \%)$ & I (5\%) & 27 (II\%) & 0.029 & $\mathrm{~B}>\mathrm{U}^{*}, \mathrm{C}^{*}$ \\
\hline \multicolumn{6}{|l|}{ Ideation } \\
\hline $\operatorname{Lv} 2>0$ & $6(30 \%)$ & $2(10 \%)$ & $24(10 \%)$ & 0.025 & $\mathrm{~B}^{*}>\mathrm{C}$ \\
\hline \multicolumn{6}{|c|}{ Self perception and interpersonal perception } \\
\hline SumT $=0$ & 19 (95\%) & $19(95 \%)$ & 134 (56\%) & 0.000 & $\mathrm{~B}^{*}, \mathrm{U}^{*}>\mathrm{C}$ \\
\hline $\mathrm{COP}=0$ & $9(45 \%)$ & I 4 (70\%) & 89 (37\%) & 0.014 & $\mathrm{U}^{*}>\mathrm{C}$ \\
\hline
\end{tabular}

Notes: The following variables were not statistically significant between groups: (Controls) Introversive, Ambitent, Pervasive Extratensive, Pervasive Introversive, Avoidant, D Score $>0$, D Score = 0, D Score $<0$, D Score $<-$ I, Adj D Score $>0$, Adj D Score = 0, Adj D Score $<0$, Adj D Score $<-1$, R $<17$, R $>27$, FM + m $<$ Sum Shd; (Processing) Zd $>+3.0$, Zd $<-3.0, \mathrm{DQv}>2$; (Mediation) XA\% $>0.89, \mathrm{XA} \%<0.70$, WDA\% $<0.75, \mathrm{X}+\%<0.55, \mathrm{X}+\%>0.20, \mathrm{X}-\%>0.20, \mathrm{X}-\%>0.30, \mathrm{Populars}<4$, $\mathrm{P}>\mathrm{a}+\mathrm{I}, \mathrm{Mp}>\mathrm{Ma}, \mathrm{MOR}>2$; (Affect) FC $>(\mathrm{CF}+\mathrm{C})+2$, FC $>(\mathrm{CF}+\mathrm{C})+\mathrm{I}$, (CF + C) $>$ FC + I, S $>2$, PureC $>0$, Afr $<0.40$, Afr $<0.50$; (Self-perception and interpersonal perception) Ego. Index $<0.33$, Ego. Index $>0.44, \mathrm{Fr}+\mathrm{rF}>0$, SumT $\geq \mathrm{I}, \mathrm{COP}>2, \mathrm{AG}=0, \mathrm{AG}>2$, GHR $>\mathrm{PHR}, \mathrm{PureH}=0$, PureH $<2$. C: a determinant reflecting use of only color in generating the response. CF: a determinant reflecting the emphasis of color over form in generating the response. FC: a determinant reflecting the emphasis of form over color in generating the response. WDA\%: a form quality calculation derived by adding FQ+, FQo, and FQu for W (Whole) and D (Detail) locations divided by the total number of $W+D$ responses. A high score indicates that form quality for whole and detail locations is appropriate. Populars: a response that occurs with unusually high frequency, at least once in every three protocols. Lv2: Level 2 Special Score; assigned to Cognitive Special Scores (Table 3) that contain a bizarre or severe quality. H: Content code for reference to a real, whole human figure. SumT: All of the Texture determinants in a record. COP: Cooperative Movement, a score reflecting the use of movement $(\mathrm{M}, \mathrm{FM}, \mathrm{m})$ that is positive or collaborative. ${ }^{* *} \mathrm{P}<0.01$; ${ }^{*} \mathrm{P}<0.05$. Adapted with permission Glossary of Comprehensive System Scores. J Pers Assess. 2007;89 Suppl I:S217-S220.22

Abbreviations: B, BD, bipolar depression; U, UD, unipolar depression; C, control. 
Table 5 Odds ratios for a bipolar or unipolar diagnosis according to Rorschach variables and other features

\begin{tabular}{|c|c|c|}
\hline $\begin{array}{l}\text { Rorschach } \\
\text { variables and } \\
\text { other features }\end{array}$ & $\begin{array}{l}\text { Univariate analysis: } \\
\text { OR }(95 \% \mathrm{CI}) \\
P \text { value }\end{array}$ & $\begin{array}{l}\text { Multivariate } \\
\text { analysis: OR } \\
(95 \% \mathrm{CI}), P \text { value }\end{array}$ \\
\hline Age & 0.97 (0.93-I.0I), 0.15 & Not done \\
\hline Gender & 0.54 (0.15-1.92), 0.339 & Not done \\
\hline $\begin{array}{l}\text { Period from onset } \\
\text { to Rorschach }\end{array}$ & I.38 (I.08-I.77), 0.009** & $\begin{array}{l}1.40(1.09-1.81), \\
0.009 * *\end{array}$ \\
\hline $\mathrm{R}$ & 1.05 (0.98-I.I2), 0.190 & Not done \\
\hline (CF & 6.00 (1.08-33.27), 0.040* & $\begin{array}{l}8.61(1.28-58.02) \\
0.027^{*}\end{array}$ \\
\hline Populars $>7$ & 8.14 (0.88-75.48), 0.065 & Not done \\
\hline PureC $>$ I & 97787 (0- very large), 0.876 & Not done \\
\hline $\mathrm{DR} 2>0$ & 8.14 (0.88-75.48), 0.065 & Not done \\
\hline WSum6 & 1.07 (I.00-I.I5), 0.054 & Not done \\
\hline
\end{tabular}

Notes: $* * P<0.01 ; * P<0.05$. Values calculated by multivariate logistic regression analysis using variables that showed statistical significance in univariate analysis as independent variables.

Abbreviations: $\mathrm{Cl}$, confidence interval; OR, odds ratio.

the control group (Table 2). These special cognitive scores indicate specific verbal expressions, such as deviant verbalization, inappropriate combinations, deviant response, and inappropriate logic. WSum6 expresses the severity of thought disorder and cognitive slippage, as well as primary process thinking. ${ }^{13,16,21}$ As Table 3 shows, DR2 $>0$ (deviant responses, level 2) was significantly more frequent in the bipolar depression group than in the unipolar depression and control groups. Level 2 responses for special scores indicate that inappropriate thought or deviation from the theme is more marked. ${ }^{16}$ Other special scores (DV1 and DR1) were more frequent in the bipolar depression group than in the control group (Table 3). Consequently, more frequent DR2 responses in conjunction with DV1 and DR1 responses contributed to the higher WSum6 scores in the bipolar depression group than in other groups.

In the affect cluster, two scores, ie, $(\mathrm{CF}+\mathrm{C})>\mathrm{FC}+2$ and PureC $>1$, were significantly more frequent in the bipolar depression group than in the other groups (Table 4). In fact, $\mathrm{C}$ codes a determinant reflecting use of color in generating the response, $\mathrm{CF}$ codes a determinant reflecting emphasis of color over form in generating the response, and FC codes a determinant reflecting emphasis of form over color in generating the response..$^{22}$ Consequently, control of the color response was laxer in the bipolar depression group. Another score, ie, Blends, was lower in the unipolar depression group, but this score did not differ between the two depressed groups (Table 2).

In the mediation cluster, Populars $>7$ was significantly more frequent in the bipolar depression group than in the other groups (Table 4). Populars is a response that occurs with unusually high frequency, at least once in every three protocols. ${ }^{22}$ Other scores, ie, FQo and WDA $\%<0.85$, were less frequent in the unipolar depression group and more frequent in the bipolar depression group, but these scores did not differ between the two depressed groups (Tables 2 and 4).

In other clusters (controls, processing and self-perception, and interpersonal perception), some scores differed between the bipolar depression or unipolar depression group and the control group, but did not differ between the two depressed groups (Tables 2 and 4 ).

To control for potential confounders, such as gender, age, years from onset of the illness to Rorschach testing, or R (number of responses to the cards), we performed a logistic regression analysis. The results of univariate and multivariate analysis are shown in Table 5. Univariate logistic regression analysis was used to screen variables that were significantly associated with a bipolar diagnosis. Selected variables were clinical features (age, gender, period from onset of illness to Rorschach test), R, and Rorschach variables that had shown significant differences between bipolar and unipolar depression. Periods from onset of illness to Rorschach testing and $(\mathrm{CF}+\mathrm{C})>\mathrm{FC}+2$ were positive by univariate logistic regression analysis, but other factors were negative (Table 5). Multivariate logistic regression analysis was performed using two variables that were significant in univariate analysis to identify independent factors for a bipolar or unipolar diagnosis and to control for confounding and interactions. $(\mathrm{CF}+\mathrm{C})>\mathrm{FC}+2$ was revealed to be an independent predictor of a bipolar diagnosis.

\section{Discussion}

The main finding of this study is that the bipolar depression group had significantly higher scores or positive findings in five variables of the Rorschach test, ie, WSum6, DR2 $>0$, $(\mathrm{CF}+\mathrm{C})>\mathrm{FC}+2$, PureC $>1$, and Populars $>7$, as assessed using the Comprehensive System, ${ }^{16}$ than the unipolar depression group. These scores were also significantly different between the bipolar depression group and healthy control group, suggesting that the positive findings or higher scores observed in patients with bipolar depression are aberrant. The time interval between onset of illness to the final observation was, on average, 9.3 years or longer in both the bipolar depression and unipolar depression groups, and was of adequate duration to confirm the differential diagnosis of bipolar and unipolar depression. Therefore, it is less likely that the unipolar depression group included false unipolar depression, ie, latent bipolar disorder. The results of this 
study indicate the specific findings for bipolar depression in the Rorschach test.

The WSum6 scores, which reflect cognitive slippage or thought disorder, were not different between the unipolar depression and control groups, but were higher in the bipolar depression group than in the other groups. The bipolar depression group showed a higher frequency of DR2 (deviant response, level 2) among these special scores than did other groups. Actually, DR2 represents an inappropriate phrase and circumstantiality that is bizarre or outside the bounds of reality, and also indicates thought disorder by which subjects stray from a task before them. ${ }^{22}$ Deviant response is often observed in affective disorders and suggests severe dysfunction. ${ }^{15}$ The Rorschach test is a sensitive instrument for detecting mild thought disorders that cannot be detected by interviews. Thought disorder and cognitive slippage are important for understanding of the psychopathology of bipolar depression.

Singer and Brabender also reported that WSum6 scores were higher in cases of bipolar depression than in cases of unipolar depression, and that bipolar mania showed the highest scores. ${ }^{13}$ Some differences are apparent between their studies and our own, ie, DR1 and INC1 among special scores included in the WSum6 were more frequent in the bipolar depression group than in the unipolar depression group in their study, but only DR2 was more frequent in ours. In their study, DR2 was more frequent in bipolar mania than in bipolar and unipolar depression. However, it was difficult to determine whether bipolar depression and unipolar depression were aberrant or not because these findings were not compared with those in healthy controls. On the other hand, Osher et al reported higher WSum6 scores in euthymic bipolar patients and healthy children of bipolar parents than in healthy controls. ${ }^{21,23}$ They suggested that thought disorder detected by the Rorschach test might be a psychological marker or endophenotype. Unfortunately, because they did not compare bipolar depression with unipolar depression, it was not clear whether high WSum6 scores are specific for bipolar depression. Taken together with our results, WSum6 scores are high in either a manic, depressive, or euthymic state of bipolar disorder and might be a psychological marker that can differentiate between bipolar depression and unipolar depression.

Patients who had $(\mathrm{CF}+\mathrm{C})>\mathrm{FC}+2$ or PureC $>1$ and those who had Populars $>7$ were observed more often in the bipolar depression group than in other groups. According to Exner's interpretation, the former show a laxness in modulating emotion, and the latter show an orientation toward oversimplification and correctness. ${ }^{15}$ These findings were not observed in a previous study of bipolar depression and mania, ${ }^{13}$ or examined in previous studies of bipolar disorder patients and their children, ${ }^{21,23}$ although healthy offspring of bipolar parents showed lower FC scores, which might result in more $(\mathrm{CF}+\mathrm{C})>\mathrm{FC}+2$. Univariate and multivariate logistic regression analysis showed that $(\mathrm{CF}+\mathrm{C})>\mathrm{FC}+2$ was as an independent predictor of a bipolar diagnosis. Because the small sample size in this study limits the possible number of independent variables in logistic regression analysis, future studies with a larger sample size are necessary to identify independent predictors among several variables. An earlier study by Donnelly et al showed that primary response to color is observed in bipolar depression but not in unipolar depression. ${ }^{14}$ Because their study did not use the Comprehensive System, comparison with our study is difficult, but their study might point to a similar phenomenon, and probably a more PureC response. More Populars $>7$ and DR2 $>0$ seen in the bipolar depression group seems to be counterintuitive. DR2 is defined as an inappropriate phrase and circumstantiality that is bizarre or outside the bounds of reality, but is not always bizarre. ${ }^{16,22}$ DR2 may include responses associated with distractibility and flight of ideas that are diagnostic for (hypo)manic episodes and are often observed, not only in (hypo)manic episodes but also in bipolar depressive episodes. ${ }^{19}$ Thus, more Populars $>7$ reflects conventional cognition or perception and seems to be able to coexist with more DR $>2$.

Only slight differences were observed in psychiatric symptoms between bipolar and unipolar depression. ${ }^{6,24}$ Therefore, it can be difficult to differentiate bipolar depression from unipolar depression by psychiatric interview only. A personal history of manic or hypomanic episodes and a family history of bipolar disorder in a first-degree relative are useful for the differential diagnosis, but cannot differentiate unrecognized latent bipolar depression, ie, without a history of manic or hypomanic episodes, from unipolar depression. The Rorschach test is a psychological instrument that needs no special facilities. It is clinically useful and can be used easily for the differential diagnosis of bipolar and unipolar depression, as reported in this study.

Limitations to the present findings of our study include a small sample size and the use of Japanese control data from another study. ${ }^{18}$ Recruitment of local, matched nonclinical subjects as a control group is needed. A further limitation is that we did not recruit and test subjects for the Rorschach test prospectively. Using charts from our department, 
we retrospectively investigated patients who had already undergone Rorschach testing and had been followed up for a long period. Test-retesting and assessment of mood/cognitive factors are fundamental for chronic, relapsing disorders such as bipolar and unipolar depression. Further, a difference was found between depressed patients and healthy controls in terms of their average age. It is unclear whether age affects the Rorschach data in adults, and this effect has not been reported. Future studies should use a larger sample size and a prospective study design for comparison with age-matched controls and should examine Rorschach test-retesting and include more precise assessment of mood/cognitive factors that can influence the Rorschach test to evaluate the overtime stability of scores and to what extent the contingent psychological condition affects the results of Rorschach testing. Finally, as described in the Introduction, the validity of the Rorschach test has been debated for decades. A recent systematic review showed that the strength of the validity evidence of most variables in the Comprehensive System, including WSum6, DR2, $(\mathrm{CF}+\mathrm{C})>\mathrm{FC}+2$, and Populars, is excellent or good, but that of some variables, such as PureC, is low. ${ }^{25}$ Therefore, the validity of each variable is heterogeneous and this heterogeneity must be noted by quoting a recent systematic review. ${ }^{25}$

\section{Conclusion}

Our study showed thought disorder or cognitive slippage and marked laxness in modulating emotion, ie, strong emotional expression in bipolar depression. These are not usually observed in unipolar depression or in healthy controls. Taken together with previous findings, our results indicate the psychopathological characteristics of bipolar disorder. Consequently, the Rorschach test might be useful for diagnosis of unipolar depression and false unipolar depression, ie, unrecognized bipolar depression, which is a salient issue in modern psychiatry. The predictive value of the Rorschach test for this purpose warrants further investigation.

\section{Acknowledgments}

This study was partly supported by "Integrated Research on Neuropsychiatric Disorders" carried out under the Strategic Research Program for Brain Sciences by the Ministry of Education, Culture, Sports, Science and Technology of Japan, research grant 24-2 for Nervous and Mental Disorders from the Ministry of Health, Labour and Welfare, Japan, and a grant for Interdisciplinary Project for Psychosomatological Research at Hokkaido University.

\section{Disclosure}

The authors declare that they have no conflict of interest in this work.

\section{References}

1. Daban C, Colom F, Sanchez-Moreno J, García-Amador M, Vieta E. Clinical correlates of first-episode polarity in bipolar disorder. Compr Psychiatry. 2006;47:433-437.

2. Akiskal HS, Maser JD, Zeller PJ, et al. Switching from 'unipolar' to bipolar II. An 11-year prospective study of clinical and temperamental predictors in 559 patients. Arch Gen Psychiatry. 1995;52:114-123.

3. Lam RW, Kennedy SH, Grigoriadis S, et al. Canadian Network for Mood and Anxiety Treatments (CANMAT) clinical guidelines for the management of major depressive disorder in adults. III. Pharmacotherapy. J Affect Disord. 2009;117:S26-S43.

4. Yatham LN, Kennedy SH, Schaffer A, et al. Canadian Network for Mood and Anxiety Treatments (CANMAT) and International Society for Bipolar Disorders (ISBD) collaborative update of CANMAT guidelines for the management of patients with bipolar disorder: update 2009. Bipolar Disord. 2009;11:225-255.

5. Tränkner A, Sander C, Schönknecht P. A critical review of the recent literature and selected therapy guidelines since 2006 on the use of lamotrigine in bipolar disorder. Neuropsychiatr Dis Treat. 2013;9: 101-111.

6. Goodwin FK, Jamison KR. Manic-Depressive Illness: Bipolar Disorders and Recurrent Depression, 2nd ed. New York, NY: Oxford University Press; 2007.

7. Suzuki K, Kusumi I, Sasaki Y, Koyama T. Serotonin-induced platelet intracellular calcium mobilization in various psychiatric disorders: is it specific to bipolar disorder? J Affect Disord. 2001;64:291-296.

8. Kameyama M, Fukuda M, Yamagishi Y, et al. Frontal lobe function in bipolar disorder: a multichannel near-infrared spectroscopy study. Neuroimage. 2006;29:172-184.

9. Donnelly EF, Murphy DL, Goodwin FK. Cross-sectional and longitudinal comparisons of bipolar and unipolar depressed groups on the MMPI. J Consult Clin Psychol. 1976;44:233-237.

10. Mendlowicz MV, Akiskal HS, Kelsoe JR, Rapaport MH, Jean-Louis G, Gillin JC. Temperament in the clinical differentiation of depressed bipolar and unipolar major depressive patients. $J$ Affect Disord. 2005;84:219-223.

11. Clarkin JF, Howieson DB, McClough J. The role of psychiatric measures in assessment and treatment. In: Hales RE, Yudofsky SC, Gabbard GO, editors. The American Psychiatric Publishing Textbook of Clinical Psychiatry, 5th ed. Washington, DC: American Psychiatric Publishing Inc; 2009

12. Garb HN, Wood JM, Nezworski MT, Grove WM, Stejskal WJ. Toward a resolution of the Rorschach controversy. Psychol Assess. 2001;13: 433-448.

13. Singer HK, Brabender V. The use of the Rorschach to differentiate unipolar and bipolar disorders. J Pers Assess. 1993;60:333-345.

14. Donnelly EF, Murphy DL, Scott WH. Perception and cognition in patients with bipolar and unipolar depressive disorders. A study in Rorschach responding. Arch Gen Psychiatry. 1975;32:1128-1131.

15. Exner J Jr. The Rorschach, Basic Foundations and Principles of Interpretation, 4th ed. Hoboken, NY: John Wiley \& Sons; 2003:1.

16. Exner J Jr. A Rorschach Workbook for the Comprehensive System, 5th ed. Asheville, NC: Rorschach Workshops; 2001.

17. Exner J Jr. A Rorschach Workbook for the Comprehensive System, 4th ed. Asheville, NC: Rorschach Workshops; 1995.

18. Nakamura N, Fuchigami Y, Tsugawa R. Rorschach Comprehensive System data for a sample of 240 adult nonpatients from Japan. J Pers Assess. 2007;89 Suppl 1:S97-S102.

19. American Psychiatric Association. Diagnostic and Statistical Manual of Mental Disorders: DSM-IV. Washington, DC: American Psychiatric Association; 1994. 
20. Ghaemi S, Ko J, Goodwin F. The bipolar spectrum and the antidepressant view of the world. J Psychiatr Pract. 2001;7:287-297.

21. Osher Y, Bersudsky Y. Thought disorder in euthymic bipolar patients: a possible endophenotype of bipolar affective disorder? J Nerv Ment Dis. 2007;195:857-860.

22. Glossary of Comprehensive System Scores. J Pers Assess. 2007;89 Suppl 1:S217-S220.

23. Osher Y, Mandel B, Shapiro E, Belmaker RH. Rorschach markers in offspring of manic-depressive patients. J Affect Disord. 2000;59: 231-236.
24. Kiejna A, Rymaszewska J, Hadryś T, Suwalska A, Łojko D, Rybakowski JK. Bipolar or unipolar? The question for clinicians and researchers. J Affect Disord. 2006;93:177-183.

25. Mihura JL, Meyer GJ, Dumitrascu N, Bombel G. The validity of individual Rorschach variables: systematic reviews and meta-analyses of the Comprehensive System. Psychol Bull. August 27, 2012. [Epub ahead of print.]

\section{Publish your work in this journal}

Neuropsychiatric Disease and Treatment is an international, peerreviewed journal of clinical therapeutics and pharmacology focusing on concise rapid reporting of clinical or pre-clinical studies on a range of neuropsychiatric and neurological disorders. This journa is indexed on PubMed Central, the 'PsycINFO' database and CAS
The manuscript management system is completely online and includes a very quick and fair peer-review system, which is all easy to use. Visit http://www.dovepress.com/testimonials.php to read real quotes from published authors.

Submit your manuscript here: http://www.dovepress.com/neuropsychiatric-disease-and-treatment-journal 